\title{
Neutral and charged excitons in a CdTe-based quantum well
}

\author{
J.A. Gaj ${ }^{1}$, P. Kossacki ${ }^{1,2}$, P. Płochocka ${ }^{1}$, W. Maślana ${ }^{1,2}$, J. Cibert ${ }^{2}$, \\ S. Tatarenko ${ }^{2}$, and C. Radzewicz ${ }^{1}$ \\ ${ }^{1}$ Institute of Experimental Physics, Warsaw University, Hoża 69, 00-681, Warsaz, Poland \\ E-mail: Jan.Gaj@fuw.edu.pl \\ ${ }^{2}$ Laboratoire de Spectrométrie Physique, CNRS et Université Joseph Fourier-Grenoble \\ B.P. 87, 38402 Saint Martin d'Héres Cedex, France
}

Received July 12, 2004

\begin{abstract}
We present a summary of our spectroscopic studies of the oscillator strength of transitions related to the formation of neutral and positively charged excitons in modulation $p$-doped CdTe-based quantum wells. The hole concentration was controlled in the range from $10^{10} \mathrm{~cm}^{-2}$ to $10^{11} \mathrm{~cm}^{-2}$. Continuous-wave and time-resolved femtosecond pump-probe absorption measurements were performed. They allowed us to study the interacting system of excitons, trions, and free holes. Characteristic times of the system were determined, such as the trion formation time. A new explanation of so-called oscillator strength «stealing» has been proposed, in terms of spin-dependent screening. Experimental evidence is presented for optical creation of transient spin polarization in the quantum well.
\end{abstract}

PACS: 71.35.-y

\section{Introduction}

The existence of charged excitons (trions) in semiconductors was predicted by Lampert [1] already in the fifties. However, because of a very small dissociation energy, charged excitons were extremely difficult to observe in bulk crystals. A very different situation occurs in doped semiconductor quantum wells, where the confinement leads to a strong increase of the dissociation energy and charged excitons can be easily observed. The first experimental identification of charged excitons in a semiconductor quantum well by Kheng et al. [2] opened the field of experimental studies of those complexes. In particular, optical properties of both $p$ - and $n$-doped CdTe-based quantum well were extensively studied [3-6]. Transmission, photoluminescence, four wave mixing and time resolved absorption experiments were performed. Negatively $\left(\mathrm{X}^{-}\right)$and positively charged $\left(\mathrm{X}^{+}\right)$excitons are frequently observed in absorption and often dominate the excitonic photoluminescence of doped quantum wells (both in III-V and II-VI semiconductor systems) $[7,8]$. They appear as absorption or photoluminescence lines a few meV below neutral exciton transition.
In this paper we present a summary of our studies of positively charged excitons in modulation $p$-doped CdTe-based quantum wells. We had a possibility to control the hole concentration by additional illumination in the range up to $10^{11} \mathrm{~cm}^{-2}$. Thus relative intensity of charged and neutral excitons was controlled. Therefore we were able to investigate the role of the hole gas and the interplay between the charged- and the neutral excitons.

\section{Experiments}

The experimental results were obtained on modulation doped structures consisting of a single $80 \AA$ quantum well ( $\mathrm{QW})$ of $\mathrm{Cd}_{1-x} \mathrm{Mn}_{x} \mathrm{Te}(x \approx 0.0018)$ embedded between $\mathrm{Cd}_{0.66} \mathrm{Zn}_{0.07} \mathrm{Mg}_{0.27} \mathrm{Te}$ barriers grown pseudomorphically on a $(100) \mathrm{Cd}_{0.88} \mathrm{Zn}_{0.12} \mathrm{Te}$ substrate. Due to strain and confinement the energy separation between heavy and light hole excitons is larger than $15 \mathrm{meV}$. Therefore only heavy hole excitons were observed in our experiments. Modulation $p$-type doping was assured by a nitrogen-doped layer placed at $200 \AA$ from the QW. The density of the hole gas in the QW was controlled by an additional illumination with photon energy above the gap of the barriers, pro- 
vided by a tungsten halogen lamp with a blue filter: the control mechanism and its calibration are described in detail in [9].

The continuous wave $(\mathrm{cw})$ transmission through the sample was measured as a function of hole gas density. The external magnetic field was applied in a Faraday configuration. We also measured time resolved absorption in a pump-probe configuration. The pulses were generated by a $\mathrm{Ti}^{3+}: \mathrm{Al}_{2} \mathrm{O}_{3}$ laser tuned to $765 \mathrm{~nm}(1620 \mathrm{meV})$, at a repetition rate of $100 \mathrm{MHz}$. The duration of the laser pulse was about $100 \mathrm{fs}$ and the spectral width about $40 \mathrm{~nm}(80 \mathrm{meV})$, which is much broader than the separation between the neutral exciton line $\mathrm{X}$ and the charged exciton line $\mathrm{X}^{+}$. To excite only one transition (charged or neutral exciton) the pump pulse was shaped to a spectral width less than $1 \mathrm{~nm}$ and a duration of about $2 \mathrm{ps}$, thus creating either $\mathrm{X}$, or $\mathrm{X}^{+}$by the binding of an additional hole. We analyzed the dynamics of both transitions by a spectrally broad probe pulse. The pump and probe pulses were focused on the sample to a common spot of diameter smaller than $100 \mu \mathrm{m}$, and the spectrum of the pump pulse transmitted through the sample was recorded as a function of the pump-probe delay. The power of both pulses was controlled independently, the pump-to-probe intensity ratio being at least 20:1. The average power of the pump beam was typically $300 \mu \mathrm{W}$, which results in the creation of a few times $10^{10} \mathrm{~cm}^{-2}$ excitons by each pulse. The pump beam was polarized circularly (by convention, $\sigma^{+}$, creating electrons with spin $-1 / 2$ in the conduction band, and holes of momentum $+3 / 2$ in the $-3 / 2$ spin-down valence subband, Fig. 1). The probe beam was detected behind the sample in both circular polarizations, measuring the absorption associated with the creation of either $\mathrm{X}$ or $\mathrm{X}^{+}$having electron-hole pairs of the same

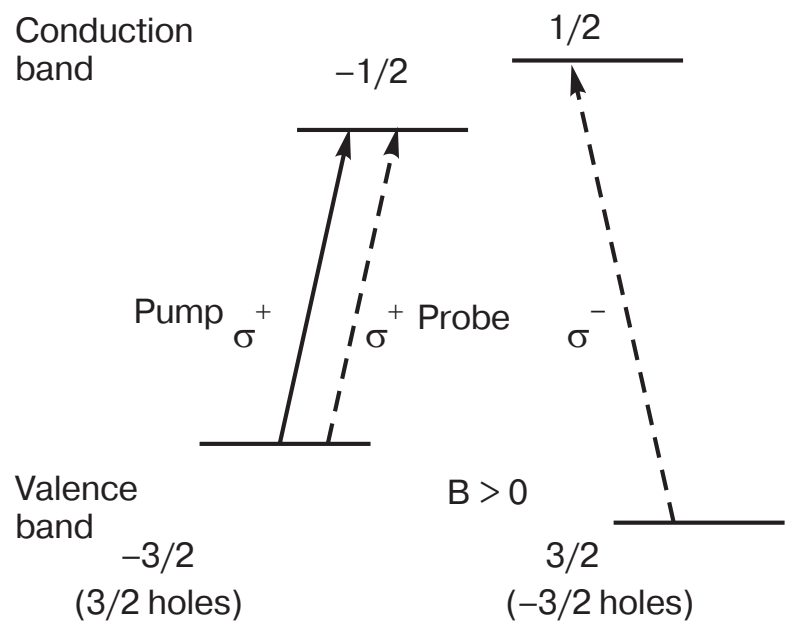

Fig. 1. Optical transitions for pump and probe pulses in magnetic field $B>0$. spin as the pump $\left(\sigma^{+}\right.$, co-polarized $)$, or opposite $\left(\sigma^{-}\right.$, cross-polarized). Therefore, we could analyze the influence of the charged exciton on the neutral exciton and separate spin-dependent contribution by separation of the light polarization. Additionally, by applying magnetic field we were able to resolve all four polarization configurations: pump with $\sigma^{-}$probe with $\sigma^{+}(-/+)$and $\sigma^{-}(-/-)$and pump polarized $\sigma^{+}$and probe $\sigma^{+}(+/+)$and $\sigma^{-}(+/-)$. To analyze the neutral and charged exciton line intensities two Gaussian functions were fitted to the cw or time-resolved spectra. In some experiments difference spectra were analyzed to expose the change in the optical absorption under influence of the pump pulse.

\section{Results}

\section{Continuous wave measurements}

Let us start from results obtained in cw transmission measurements. In Fig. 2 the transmission through quantum well, measured for different hole concentrations, is shown. Two absorption lines are observed. The higher energy line is related to the neutral exciton (X). The second line, lying at lower energy, is related to the positively charged exciton $\left(\mathrm{X}^{+}\right)$. A strong influence of the hole gas on intensity of both transitions is observed. The neutral exciton intensity decreases with increasing hole concentration. Simultaneously the charged exciton intensity increases. For low hole concentrations the charged exciton intensity is proportional to the hole concentration in the relevant spin subband. However, for higher hole concentration the intensity of the charged exciton saturates, because states with higher $\mathbf{k}$ are filled.

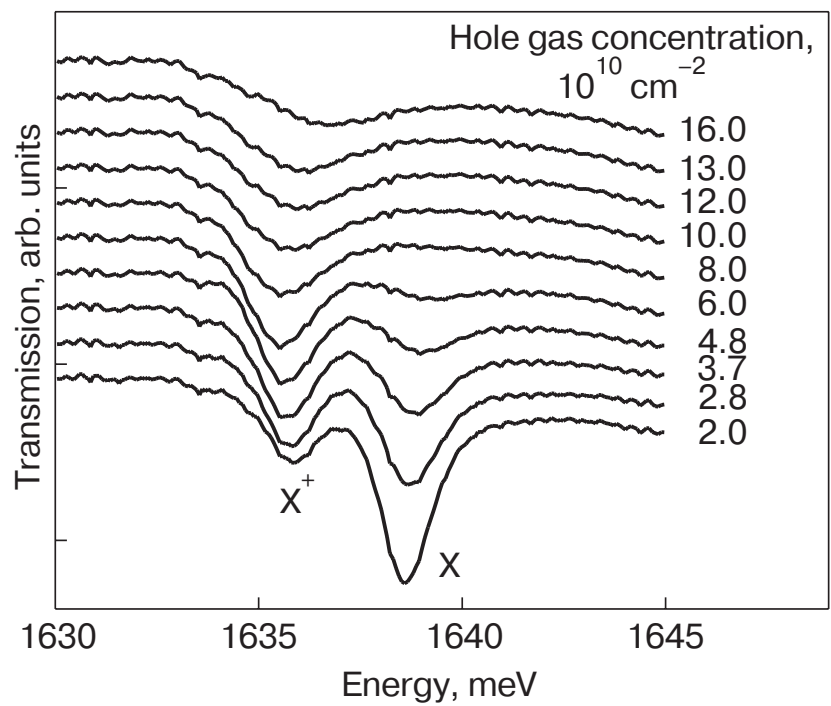

Fig. 2. Transmission through the quantum well measured for different hole concentrations. 
The changes of relative intensity of the charged and neutral exciton were observed for both negatively and positively charged excitons $[2,9,10]$. This effect was called oscillator strength stealing (OSS) from the neutral exciton by the charged exciton. However, the change of the charged exciton oscillator strength is not equal to the change of the neutral exciton oscillator strength. In this way the sum rule is not applicable; only after taking into account additional optical transitions involving scattering processes is the sum rule approximately obeyed [3,11]. We will show that it is possible to explain the reduction of the neutral exciton intensity by screening: a normal and a spin-dependent one. The two types of screening can be separated by magnetooptical experiments.

Measurements of cw optical density in external magnetic field were also performed. The selection rules for charged and neutral exciton are the same as for pump-probe experiments, presented in Fig. 1. In Fig. 3, $a$ the transmission spectra in both polarizations in magnetic field are shown. In $\sigma^{+}$polarization we observe that the neutral exciton line dominates the charged exciton line. In Fig. 3,b, where intensities of the neutral and charged exciton lines are plotted versus magnetic field, the charged exciton line completely disappears in $-0.3 \mathrm{~T}$. However, for the $\sigma^{-}$polarization the charged exciton line gets stronger with increasing magnetic field. In magnetic field as low as $-0.3 \mathrm{~T}$ the hole gas is fully polarized in one spin subband, due to the giant Zeeman effect in the semimagnetic QW. A charged exciton is formed by the a binding of an additional hole from the pre-existing gas with spin opposite to those created by the light. In one polarization trions cannot be formed - there are no pre-existing holes in the opposite spin subband. In the opposite polarization the intensity of the charged exciton dominates, while the intensity of the neutral exciton is smaller. This correlation between charged and neutral exciton intensities became known as intensity- or oscillator strength stealing. No microscopic explanation of this effect has been proposed so far. Using external magnetic field and external illumination we can control the relative number of charged and neutral excitons. An important conclusion from $\mathrm{cw}$ measurements is that the spin-dependent effects are essential. However, their exact nature was still unclear. Significant progress was obtained in time-resolved studies.

\section{Time-resolved absorption measurements}

We measured the time-resolved absorption [6] hoping to explain the origin of the oscillator strength stealing. In Fig. 4, $a$ the optical density for negative and short positive delays ( $0.5 \mathrm{ps})$ is presented. We observe two absorption lines, one related to the charged exciton, the other to the neutral exciton. At zero delay (when the strong pump pulse excites the sample) the intensity of the charged exciton decreases and inten-

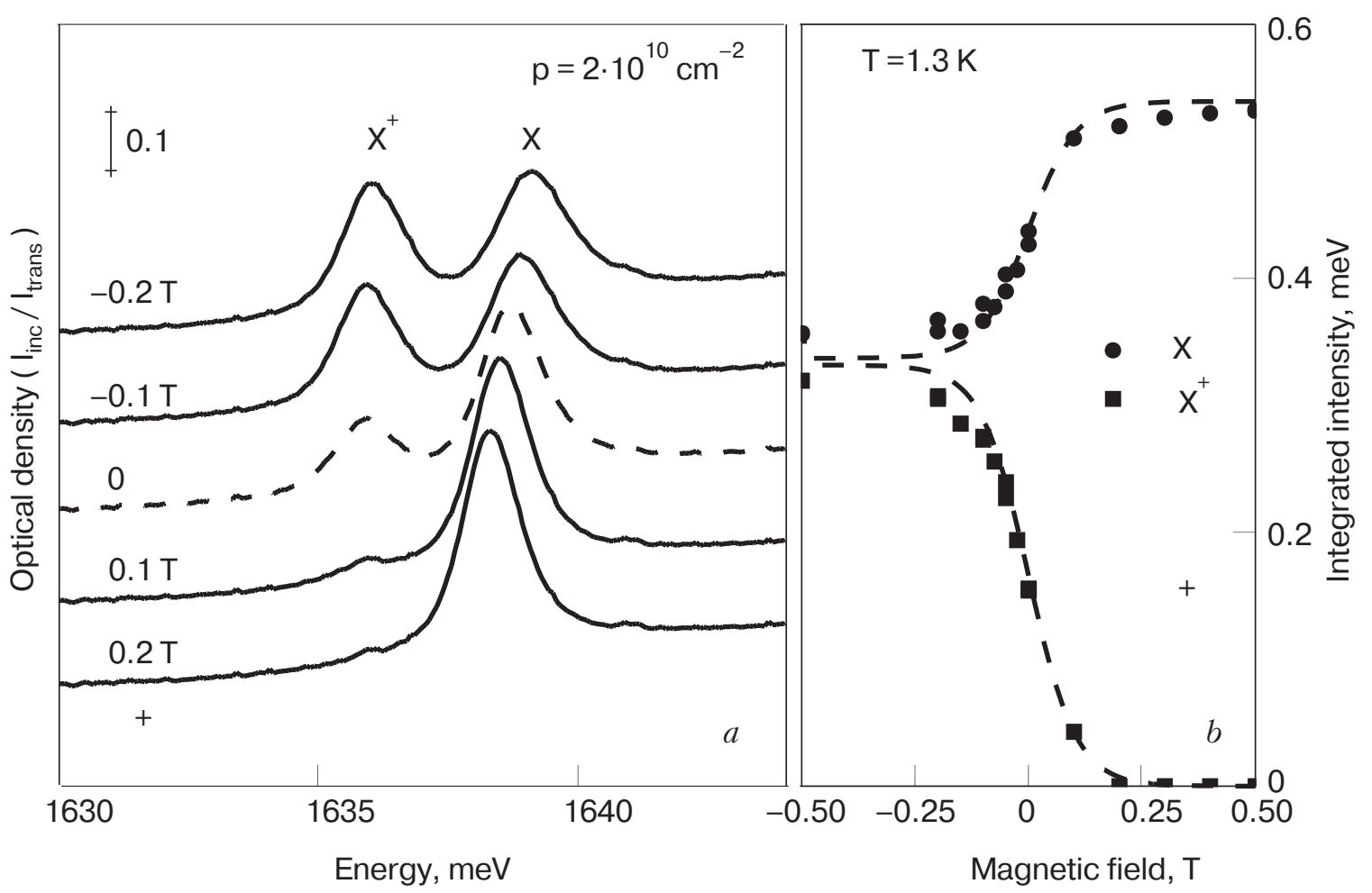

Fig. 3. Optical density of a CdMnTe quantum well in magnetic field (hole concentration was $\left.p=2 \cdot 10^{10} \mathrm{~cm}^{-2}\right)(a)$. Integrated line intensities as a function of magnetic field $(b)$. 

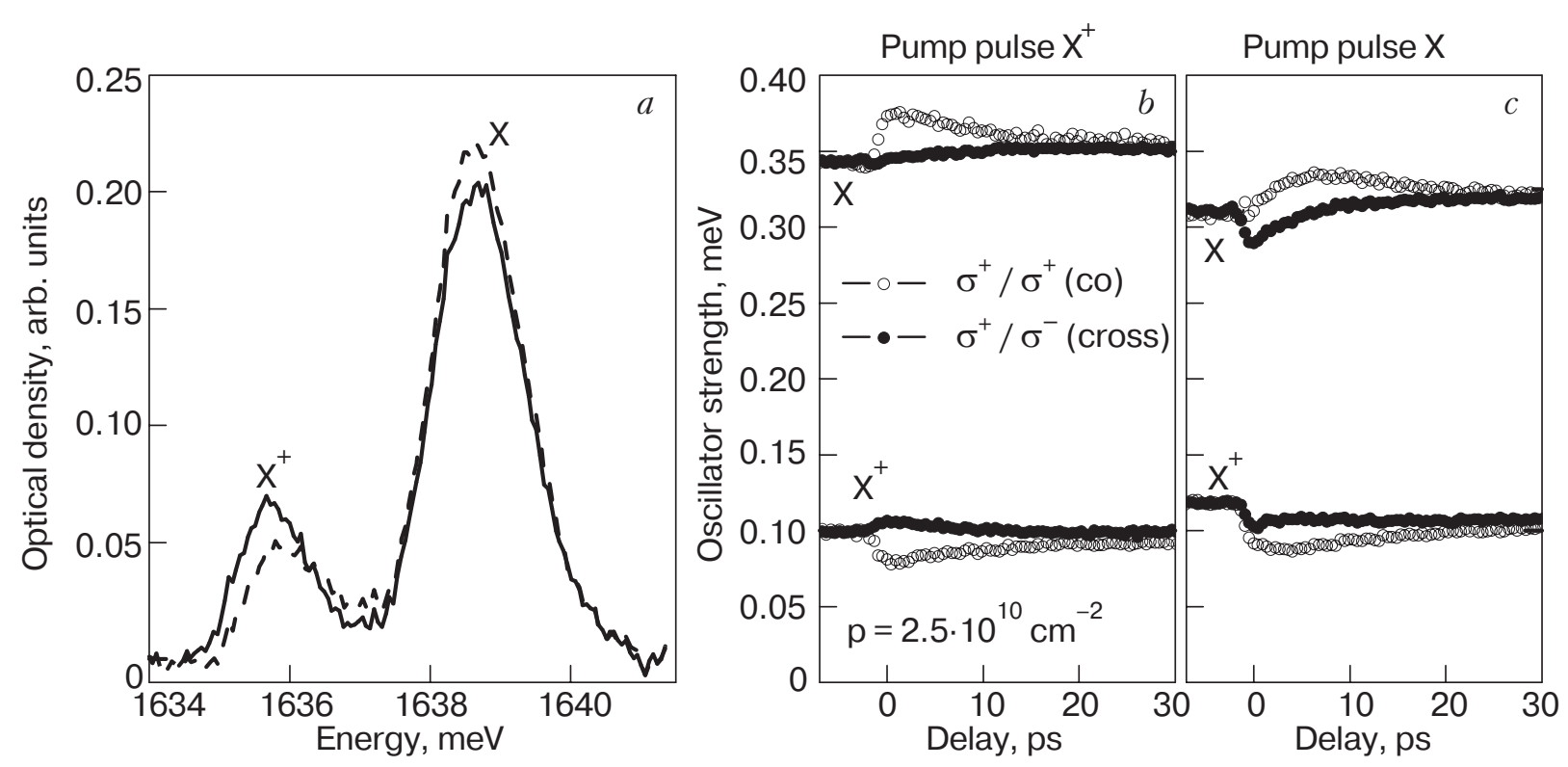

Fig. 4. Optical density for a QW with hole density $p \approx 2.5 \cdot 10^{10} \mathrm{~cm}^{-2}$, at negative delay ( $-8 \mathrm{ps}$, solid line) and short positive delays $\left(0.5 \mathrm{ps}\right.$, dashed line), for a co-polarized pump pulse tuned to $\mathrm{X}^{+}(a)$; evolution of the $\mathrm{X}_{\text {and }} \mathrm{X}^{+}$oscillator strength with pump pulse tuned to $\mathrm{X}^{+}(b)$; evolution of $\mathrm{X}$ and $\mathrm{X}^{+}$with pump pulse tuned to $\mathrm{X}(c)$.

sity of the neutral exciton increases for co-polarized pump and probe pulses. The line intensities are plotted versus pump-probe delay time on Fig. 4,b,c. Temporal evolution of the oscillator strength of the charged and neutral excitons is shown Fig. $4, b$ for coand cross-polarized beams when the pump pulse is tuned to resonance with the charged exciton. Results for pump pulse tuned to the neutral exciton are presented in Fig. 4,c.

Let us start the discussion from co-polarized beams. We observe an intensity decrease of the charged exciton, accompanied by an intensity increase of the exciton at zero delay time. This variation is faster when the pump pulse is tuned to resonance with the charged exciton; when the pump pulse is tuned to resonance with the neutral exciton, the same effects are observed with a delay. We observe the same behavior as that observed in transmission measurements (Fig. 2), with one important difference: now we do not modify the density of pre-existing holes. The only thing which changes in time-resolved measurements, is the occupation of charged exciton states. This fact led us to a new explanation of the oscillator strength stealing phenomena, in terms of spin-dependent screening. Let us assume that intensity of neutral exciton decreases due to screening by holes with spin opposite to the spin of the hole created by light. In this way we can explain why in $\mathrm{cw}$ measurements the increase of the hole density results in a decrease of neutral exciton intensity. In time-resolved results, the holes bound into the charged excitons (resonant creation of the charged excitons by the pump) cannot screen the neutral exciton any more, and its intensity increases, as shown in Fig. 4,b. The decrease of the charged exciton intensity is due to bleaching of the transition: the number of charged excitons created by the pump pulse is comparable with the number of pre-existing holes; therefore the probe pulse with the same polarization cannot form charged excitons - the transition is blocked. The conclusion is that the decrease of neutral exciton intensity is due to screening by holes with spin opposite to that of the holes involved in the neutral exciton - a spin-dependent effect. The second important conclusion is that binding holes into the charged exciton excludes them from interaction with the rest of the system, specially from screening the neutral exciton. In the case of pumping into the neutral exciton we observed the same effect, but the increase of the neutral exciton intensity is delayed. In this case the charged exciton is not formed directly. The pump creates neutral excitons, and then some time is needed to bind additional holes and form charged excitons. Therefore the increase of neutral exciton intensity, when the pump pulse is tuned to resonance with the neutral exciton, occurs with a rise time related to formation of charged excitons. We found that this rise time depends on hole concentration in following way: 5,2 , and $1 \mathrm{ps}$ for hole densities $2.5 \cdot 10^{10}, 3 \cdot 10^{10}$, and $4 \cdot 10^{10} \mathrm{~cm}^{-2}$ respectively [6]. The formation time is faster for higher hole concentration, in good agreement with the PL results presented in $[12,13]$. 
Let us turn to the situation when the pump and probe pulses are cross-polarized. We observe a completely different time evolution of charged and neutral exciton intensities depending on whether the pump pulse is tuned to resonance with the charged or neutral exciton. In Fig. 4,b (pump resonant with $\mathrm{X}^{+}$) the intensity of the charged exciton increases a little bit, and the intensity of the neutral exciton does not change in time. However, when we tune the pump pulse to resonance with neutral exciton (Fig. 4,c), the intensity of both transitions decreases at zero delay. This behavior can be explained by spin-dependent screening. When we pump into the charged exciton we bind holes. The charged exciton is sensitive to holes with both spins (because it consists of two holes with opposite spin). The bound holes are excluded from screening trions, which increase in intensity. In co-polarization this effect is masked by the bleaching discussed previously. When we pump the neutral exciton, a decrease in intensity is observed in cross-polarization for both neutral and charged excitons. We explain it by exciton-exciton interaction: screening and phase space filling.

We can use the fact that the charged exciton intensity is proportional to the density of holes in the relevant spin subband to find characteristic time of the heavy hole spin flip. Let us assume that a strong pump pulse creates a nonequilibrium population of heavy holes in one spin subband. Then the intensity of the charged exciton when the probe pulse is $\sigma^{+}$polarized is proportional to the density of holes in spin $3 / 2$ subband. For $\sigma^{-}$polarization of the probe pulse intensity of the charged exciton is proportional to the number oh heavy holes with spin $-3 / 2$. Therefore, the time evolution of the difference of intensity of charged exciton in both circular polarizations gives us information about spin relaxation of the heavy holes. In a complicated systems like ours, other spin polarizations (of excitons, trions) can also be responsible for the intensity difference. However, we believe that the hole spin polarization is predominant. In Fig. 5 the temporal evolution of the difference between $\mathrm{co}^{-}$and cross-polarized intensities of the charged exciton is plotted. The decay time is $8 \mathrm{ps}$, and we found it weakly dependent on the hole concentration in the investigated range. This value is comparable to the spin relaxation times determined for holes bound in excitons and trions. Photoluminescence experiments with much lower excitation power and very similar heterostructures gave values from about 3 ps [14] through 20 ps [12] up to 35 ps [13].

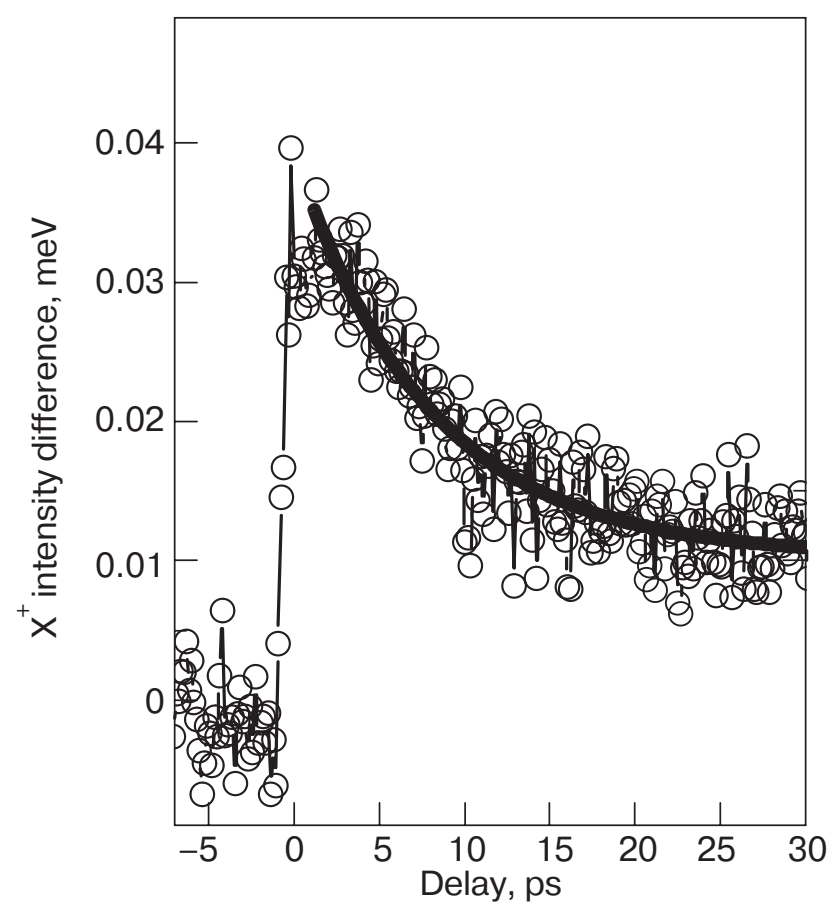

Fig. 5. Temporal evolution of difference between charged exciton oscillator strengths observed in «cross» and «co» circular polarizations without external magnetic field. Line presents fit of exponential decay with decay time 8 ps.

\section{Conclusions}

We have shown that pump-probe time-resolved absorption measurements can be a useful tool for studying interaction mechanisms in the exciton-carrier-trion system. We proposed a new explanation of the known oscillator strength stealing effect by spindependent screening. We showed experimentally a new optical creation mechanism of spin polarization of a 2D carrier gas in a semiconductor quantum well. Pump-probe absorption experiments enabled us to measure characteristic times of the excitons and carriers, such as the trion creation time or spin relaxation time.

This work has been partially supported by KBN grants 2 P03B 00225 and PBZ-KBN-044/P03/2001, and the Polonium program.

1. M.S. Lampert, Phys. Rev. Lett. 1, 450 (1958).

2. K. Kheng, R. Cox, Y. Merle d'Aubigné, F. Bassani, K. Saminadayar, and S. Tatarenko., Phys. Rev. Lett. 71, 1752 (1993).

3. R.T. Cox, R.B. Miller, K. Saminadayar, and T. Baron, Phys. Rev. B69, 235303 (2004).

4. K. Kheng, Ann. Phys. 20, 229 (1995).

5. G.V. Astakhov, D.R. Yakovlev, V.P. Kochereshko, W. Ossau, J. Nurnberger, and W. Faschinger, Phys. Rev. B60, R8485 (1999). 
6. P. Plochocka, P. Kossacki, W. Maślana, J. Cibert, S. Tatarenko, C. Radzewicz, and J.A. Gaj, Phys. Rev. Lett. 92, 177402 (2004).

7. G. Finkelstein, H. Shtrikman, and I. Bar-Joseph, Phys. Rev. Lett. 976, 74 (1995).

8. A.J. Shields, J.L. Osborn, M.Y. Simmons, M. Pepper, and D. A. Ritchie, Phys. Rev. B52, 5523 (1995).

9. P. Kossacki, J. Cibert, D. Ferrand, Y. Merle d'Aubigné, A. Arnoult, A. Wasiela, S. Tatarenko, and J.A. Gaj, Phys. Rev. B60, 16018 (1999).

10. T. Brunhes, R. André, A. Arnoult, J. Cibert, and A. Wasiela, Phys. Rev. B60, 11568 (1999).
11. A. Esser, R. Zimmermann, and E. Runge, Phys. Status Solidi B227, 317 (2001).

12. E. Vanelle, M. Paillard, X. Marie, T. Amand, P. Gilliot, D. Brinkmann, R. Lévy, J. Cibert, and S. Tatarenko, Phys. Rev. B62, 2696 (2000).

13. P. Kossacki, J. Phys. C13, R471 (2003), and references cited therein.

14. C. Camilleri, F. Teppe, D. Scalbert, Y.G. Semenov, M. Nawrocki, M. Dyakonov, J. Cibert, S. Tatarenko, and T. Wojtowicz, Phys. Rev. B64, 085331 (2001). 